\title{
PRINCIPLES OF A CODED NUMBERING SYSTEM AND ITS APPLICATION
} TO OPEN CLUSTERS

\author{
J.C. Mermilliod \\ Institut d'Astronomie de l'Université de Lausanne \\ and Observatoire de Genève, Switzerland
}

When we began to collect systematically photometric data it was felt that we needed an appropriate system of identification for our catalogues on magnetic tape. The current system of identification which uses catalogue or list name and star number is best suitable for printed catalogues but brings out many drawbacks when using and intersecting catalogues on magnetic tapes. Thus we developed a coded system of identification with the two basic conditions that it allows to include in the files stars with all possible kinds of numbering found in the literature, and that computer use was as simple as possible (Mermilliod, 1973).

One criterion of quality of a catalogue is that the stars are recorded under a single identification. Because of the exotic and complex heterogeneity in the identification of stars found in the literature, star cataloguing brings forward many problems. Due to the anarchic development of identification systems we are obliged to define priority orders among the catalogues and to alter the original designation found in the literature to give our compilation a high degree of homogeneity in the identifications. For example, photometric data for a proper motion star can be found in the literature under the following identifications: HD, DM, Gliese, Luyten LTT, Giclas G stars. Thus much secondary material is necessary in the form of transit tables and crossidentifications. This problem has to be solved separately for field stars and for stars in open clusters.

This system was first used for the photometric files prepared in the Lausanne and Geneva observatories for the CDS. 
SCHEMATIC PRINCIPLES AND MEANING OF THE CODED NUMBERS

A coded number presents 10 digits. The first is a sign which separates southern DM stars from the northern ones. For the rest of the codes, it is always positive. The general meaning of a code is contained in the second digit which runs from 0 to 9. This is the first key to decode a coded number into the two parts contained in the 8 remaining digits: a) the second key which gives the reference to a catalogue or a published list, b) the number of the star in the quoted reference. The schematic definitions of the coded numbering system are given in the appendix.

\section{SOLUTION FOR THE FIELD STARS}

Among the 73,000 measurements compiled in the UBV system (Mermilliod and Nicolet, 1976), some 46,000 concern HD, HDE and DM stars, 16,000 stars in open clusters, and 12,000 remain stars with various identifications. Some of them come from well-known catalogues, such as the Gliese catalogue (Gliese, 1969) or the luminous stars in the southern milky way (Stephenson and Sanduleak, 1971), for example. Such catalogues are very valuable and all stars contained in them having no other identification than that of a specific catalogue, are easily coded. So also are stars from the selected areas. But there are in the literature plenty of lists with less than 100 stars, or less than 1,000 for more extended survey. One good example is the Feige stars (114 in total) (Feige, 1958) and another could be a sequence of stars in a chosen constellation. Such lists have their utility too and should not be neglected during the compilation of large files. In the framework of the coded numbering system a solution to each problem has been looked for.

One major advantage of this system is that no accurate coordinates are necessary to form the files. With the references, one can easily go back to finding charts. A second one is that stars of a catalogue are recorded together in a file and not dispersed throughout it.

\section{STARS IN OPEN CLUSTERS}

Up to now, stars in open clusters were the most djfficult to include in large compilations, principally because there exist for each cluster many numbering systems. This results in an intricate situation, whose solution needs an appreciable amount of work and material. The general form of a coded number for a star in an NGC cluster is +2 , followed by the NGC number and the star number in the cluster. This is simple, but the star's number is difficult to define, due to the multiplicity of the available numbering 
systems. In order to solve correctly this problem we need crossidentification tables between different numbering systems and also between the adopted final system and the HD and DM catalogues, because bright stars in open clusters are frequently included in lists of field stars under their HD number. These realizations are explained in more detail and with references in the Catalogue of UBV photometry and MK spectral types in open clusters (Mermilliod, 1976). As for field stars, we note that stars of an open cluster are recorded all together in a specific place in a large file, and thus their usage is greatly facilitated.

CONCLUSION

Taking into account all the definitions and principles, one obtains eventually quite a homogeneous numbering system which helps to keep up to date high quality catalogues which we hope contain only a small number of redundant identifications.

Computer use has proved to be very effective and the more catalogues are coded with this system, the more easy is the comparison of many catalogues, photometric, spectroscopic and so on.

\section{REFERENCES}

Feige, J.: 1958, Astrophys. J. 128, 267. Gliese, W.: 1969, Catalogue of Nearby Stars. Mermiliiod, J.C.: 1973, CDS Intemal Report No. 5. Mermiliiod, J.C.: 1976, Astron. Astrophys. Suppl. 24, 159. Mermiliod, J.C. and Nicolet, B.: 1976, in preparation. Stephenson, C.B. and Sanduleak, N.: 1971, Publ. Warner and Swasey Obs. No. 1.

APPENDIX

Schematic definitions of the coded numbering system Durchmusterung catalogues $\pm 0.0 Z Z B B B B B \quad Z Z$ : declination zone BBBBB star number

$\mathrm{HD}$ and HDE catalogues

+1. DOBBBBBB BBBBBB star number

Qpen clusters

+2. NNNNBBBB NNNN NGC number

+3. IIIIBBBB IIII IC number

+4. AAXXBBBB

$+5 . A X X X B B B B\}$

Ananymous clusters

BBBE star number BBBB as above $A A, A$ cluster names $X X, X X$ cluster no. BBBB star number 
Star catalogues and star lists

+6. AAABBBBB

+6.1 AAABBBB

$+6.2 A A A B B B$

Selected areas

+7. AXXXBBBB A catalogue of selected areas

$X X X S . A$. number

BBBB star number

Catalogues by declination zones (except DM catalogue)

+8. AAZZBBBB AA author identification, $Z Z$ declination zone BBBB star number

Miscellaneous lists

$\begin{array}{ll}+9.0 A A A B B B B & \text { Stars in associations } \\ +9.5 R Y X X B B B & R=0 \text { : Harvard standard regions } \\ & R=1 \text { : Cousins E regions } \\ & Y=1-6, \text { stands for A-F } \\ & X X X=01-09 \text { : region designation } \\ & B B B \text { star number }\end{array}$

